\title{
Ritonavir Inhibits NF-AT Activation Through Effects on the PI-3
} Kinase/Akt Pathway Shibani Pati ${ }^{1}$, Anhthu Nguyen ${ }^{2}$, J Scott Foulke ${ }^{1}$, Frank Weichold ${ }^{3}$ and Marvin Reitz*ł1

Address: ${ }^{1}$ Institute of Human Virology, University of Maryland Biotechnology Institute, 725 W. Lombard St., Baltimore, MD 21201, ${ }^{2}$ University of Maryland, Baltimore County, Baltimore, MD 21259 and ${ }^{3}$ Morgan State University, 1700 East Cold Spring Lane, Baltimore, MD 21251

Email: Marvin Reitz* - reitz@umbi.umd.edu

* Corresponding author ‡Presenting author

from 2005 International Meeting of The Institute of Human Virology

Baltimore, USA, 29 August - 2 September 2005

Published: 8 December 2005

Retrovirology 2005, 2(Suppl I):S44 doi:10.1I86/1742-4690-2-SI-S44

The HIV protease inhibitor ritonavir has been reported to have activities unrelated to inhibition of HIV protease, including anti-tumor activity in vivo and in vitro, induction of lipodystrophy in vivo, inhibition of the 20 S proteasome, and inhibition of NFkB activation. Here we show that ritonavir also inhibits activation of NF-AT by PMA plus ionomycin and by the HHV-8 vGPCR. Inhibition of NF-AT activation occurs through the PI-3 kinase/Akt/GSK3 pathway, since ritonavir treatment leads to decreased Akt phosphorylation and a resultant decrease in GSK-3 phosphorylation. Treatment with ritonavir also inhibits the expression of NF-AT-dependent pro-inflammatory factors. Inhibition of multiple signaling pathways may help to explain the anti-tumor and other effects of ritonavir that are unrelated to its anti-retroviral activity. 